\title{
Design and Implementation of a Clinic-Based Food Pharmacy for Food Insecure, Uninsured Patients to Support Chronic Disease Self-Management
}

\author{
Marianna S. Wetherill, PhD, MPH, RDN-AP/LD ${ }^{1,2}$; Heather Chancellor McIntosh, MS, CRA ${ }^{3}$; \\ Chloe Beachy, $\mathrm{BS}^{4}$; Olivia Shadid, $\mathrm{BS}^{5}$
}

\section{INTRODUCTION}

Food insecurity, a household condition of unreliable access to an adequate, nutritious food supply, ${ }^{1}$ is increasingly recognized by the medical community for its negative implications regarding disease management outcomes. ${ }^{2,3}$ Clinic-to-community models to address food insecurity offer a direct approach for health care systems to link patients who are at risk to community food resources ${ }^{4}$ and can involve physician food prescriptions that can be filled on-site through medically tailored food pharmacies. Few published studies have described the design and implementation of food pharmacies, which may provide opportunities to address immediate food needs of patients while teaching nutrition-related disease self-management skills. The University of Oklahoma (OU) Food Pharmacy was designed to accomplish 3 outcomes: (1) improve patient knowledge and intake of affordable, medically appropriate foods, (2) reduce household food insecurity, and (3) reduce nutrition-related clinical risk factors for cardiometabolic disease.

\section{OVERVIEW}

The OU Food Pharmacy was developed as a pilot project between the Community Food Bank of Eastern Oklahoma and researchers at the OUTU School of Community Medicine and the OU College of Public Health. Tests sites were 2 OU-affiliated clinics that provided free, ongoing chronic disease management to uninsured patients, many of whom were diagnosed with hypertension, diabetes, and/or hyperlipidemia. The more important assumption of the intervention was that patients affected by food insecurity needed tailored support to enable eating behavior change, including access to healthy foods and nutrition education that considered socioeconomic resource constraints. ${ }^{5}$

\footnotetext{
${ }^{1}$ Department of Health Promotion Sciences, College of Public Health, University of Oklahoma, Tulsa, OK

${ }^{2}$ Department of Family and Community Medicine, OU-TU School of Community Medicine, University of Oklahoma, Tulsa, OK

${ }^{3}$ Office for Research Development and Scholarly Activity, School of Community Medicine, University of Oklahoma, Tulsa, OK

${ }^{4}$ Anne and Henry Zarrow School of Social Work, College of Arts and Sciences, University of Oklahoma, Tulsa, OK

${ }^{5}$ OU-TU School of Community Medicine, University of Oklahoma, Tulsa, OK

Conflict of Interest Disclosure: The authors have not stated any conflicts of interest.

Address for correspondence: Marianna S. Wetherill, PhD, MPH, RDN-AP/LD, University of Oklahoma - Tulsa Schusterman Center, College of Public Health, 4502 E 41st St, Tulsa, OK 74135; Phone: (918) 660-3684; Fax: (918) 660-3671; E-mail: marianna-wetherill@ouhsc.edu J Nutr Educ Behav. 2018;50:947-949

(C) 2018 The Authors. Published by Elsevier Inc. on behalf of Society for Nutrition Education and Behavior. This is an open access article under the CC BY-NC-ND license. (http:// creativecommons.org/licenses/by-nc-nd/4.0/)

https://doi.org/10.1016/j.jneb.2018.05.014
}

\section{Prescription Food Package}

Each food package contained fresh produce and shelf-stable foods selected by the project's dietitian in collaboration with food bank staff. Foods aligned with the Dietary Approaches to Stopping Hypertension diet, ${ }^{6}$ which was also suitable for patients with diabetes. The food package was designed to supplement a patient's monthly food supply and contained a rotation of items representing foods featured in the curriculum and recipe cards. Each shelfstable monthly package included 16-30 servings of beans, 5-16 oz nuts and seeds, $4-13$ servings of nosugar added dried fruit, 48-64 oz low-sodium vegetable juice, 14-30 servings of whole grains, $17 \mathrm{oz}$ olive oil, 7.5-14.75 oz canned fatty fish, and 1-3 anti-inflammatory spice containers $(0.65-1.1 \mathrm{oz}$ each) (Figure 1). Each box was valued at $\$ 40$ and purchased for about $\$ 24$ through the food bank's reduced-cost ordering program.

\section{Curriculum}

The Food Pharmacy curriculum included an orientation booklet that provided an overview of healthy eating principles for cardiometabolic disease, including essential information about how food choices relate to blood pressure, lipids, and blood sugar, which were further reinforced through sample low-cost, easy-to-prepare recipes that accompanied each food box. Medical student clinic interns drafted booklet content and 7 sets of recipe cards (35 in total) that were informed by the Dietary Approaches to Stopping Hypertension diet guidelines ${ }^{6}$ and illustrative of food box items. The booklet and 


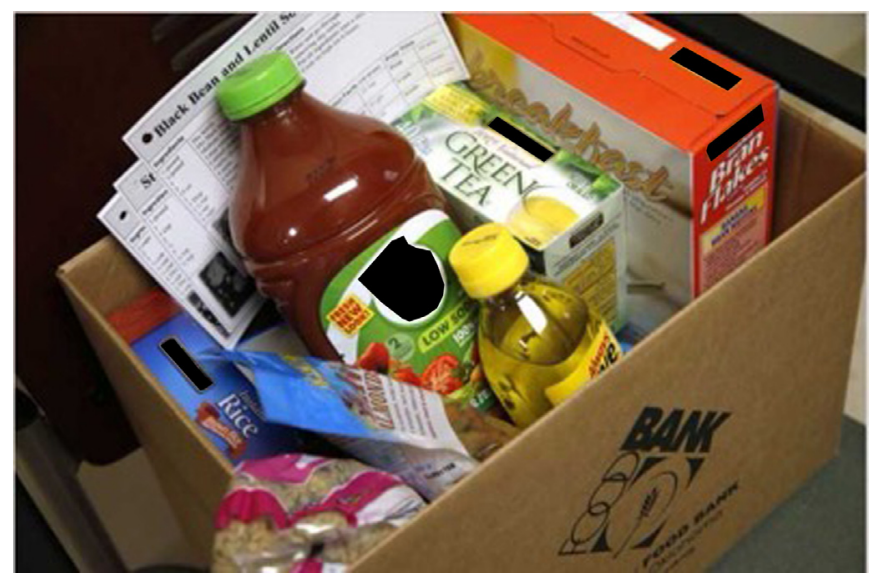

Figure 1. Example of shelf-stable monthly package from the University of Oklahoma Food Pharmacy.

recipe cards were reviewed for accuracy and further adapted for the needs of resource-limited populations by a registered dietitian with advance-practice certification in clinical nutrition and previous food banking experience. The final curriculum received additional peer review by a physician at 1 of the clinic test sites. The booklet covered 6 topics: (1) anti-inflammatory spices, herbs, and teas; (2) phytonutrients and high-potassium fruits and vegetables; (3) low-cost, plant-based protein foods; (4) whole grains; (5) healthy fats; and (6) dairy alternatives and low-fat dairy. Essential information for each topic was written at less than an eighth-grade reading level and combined with straightforward action steps, illustrative pictures, white space, and a section for patient goal setting and confidence assessment (Figure 2). Recipes were written at or below a sixth-grade reading level, pretested by medical student interns, incorporated food pharmacy foods, contained $<5-7$ ingredients, and used basic kitchen appliances and cooking techniques. Sample recipes include salmon hash, low-sodium vegetable soup, sardine patties, and lentil pasta sauce.

\section{Implementation}

Eligible participants included any patient accessing 1 of the 2 test site clinics who either self-enrolled in the program or was identified for the
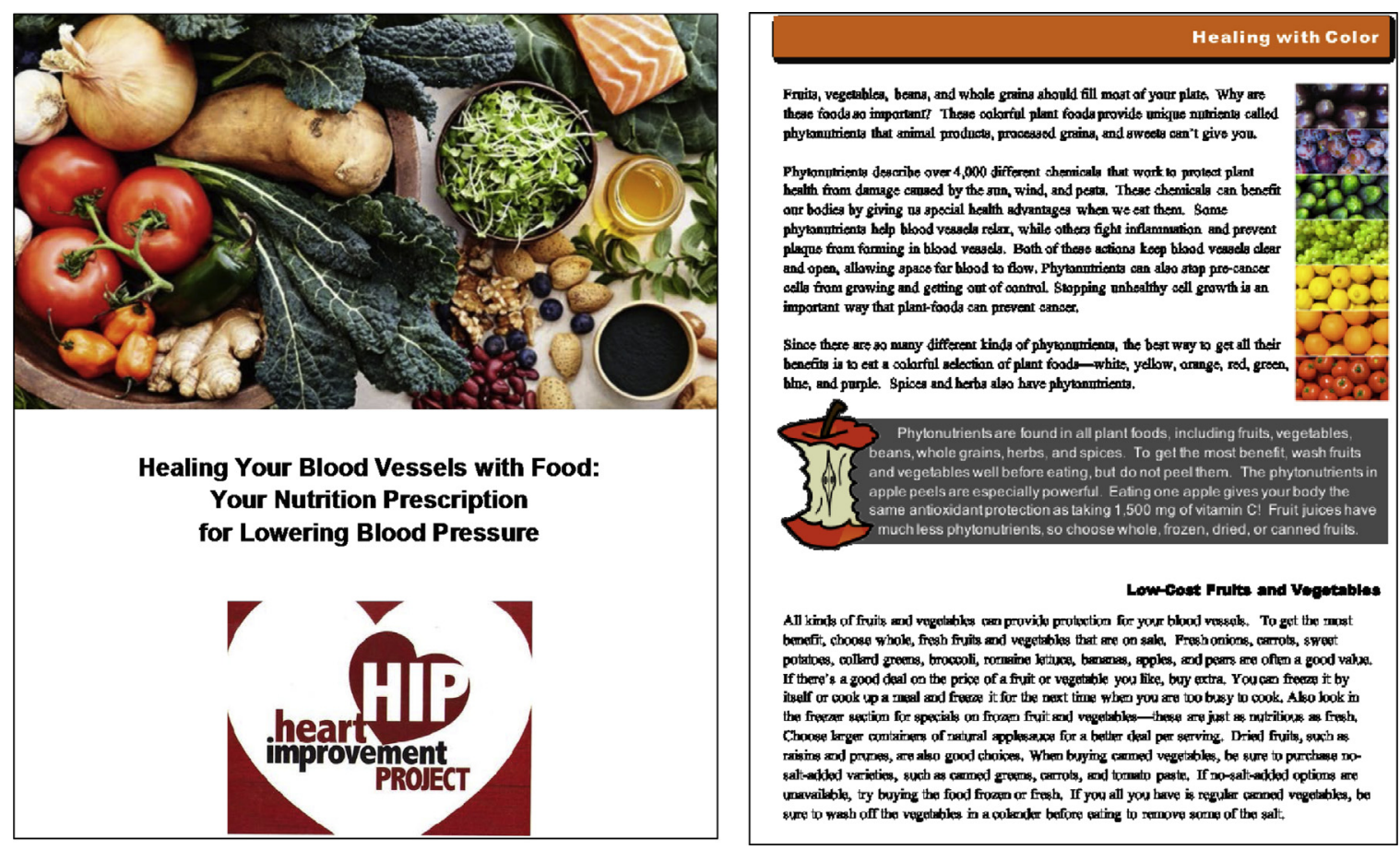

Figure 2. Excerpt from the University of Oklahoma Food Pharmacy curriculum, which included an orientation booklet with straightforward action steps, illustrative pictures, white space, and a section for patient goal setting and confidence assessment. 
program by a health care or social work provider. Upon enrollment, participants received an initial food package, the educational booklet, and 5 recipe cards during the medical appointment. Medical and social work students who received training in the curriculum by the project's dietitian oriented patients to the booklet and sample items from the food box. Participants were eligible to receive another food package during clinic hours 6 additional times with visits limited to once per month. Before each week's clinic, the project coordinator provided reminder calls to patients who were eligible for another food package. The food bank delivered shelf-stable foods monthly, which were stored on-site, and produce weekly before clinic hours for immediate distribution.

Project staff collected informal feedback from participants during each visit about their use of food box contents and recipe cards from the previous visit. For example, lowsodium vegetable juice was well received but participants strongly preferred vegetable juice in 8-oz cans instead of a 64-oz bottle. Few participants reported eating the canned sardines, so canned salmon or tuna was included in subsequent boxes.

At baseline and upon the fourth visit, the researchers administered standardized surveys to collect demographics and used validated measures of dietary intake and food security status to evaluate outcomes reported here, including the Block Fruit/ Vegetable/Fiber Screener, ${ }^{7}$ the 6-item Short-Form US Household Food Security Survey Module, ${ }^{8}$ and items from the Hunger in America 2014 Study. ${ }^{9}$ Blood pressure data were obtained through medical record review.

\section{PRELIMINARY OUTCOMES}

Rolling enrollment occurred between July, 2016 and July, $2017(\mathrm{n}=80)$. Mean age of participants was 51.7 years $(\mathrm{SD}=7.5)$. All participants had hypertension, diabetes, prediabetes, and/or hyperlipidemia. The majority of participants were female (66\%), earned <\$15,000/y (74\%), were food insecure (87\%), and reported choosing between food and medicine at least once in the past year $(67 \%)$. Slightly more than half $(55 \%)$ received benefits through the Supplemental Nutrition Assistance Program.

Longitudinal outcomes were analyzed for participants with complete data who had accessed food assistance at least 4 times $(n=43)$. Assumptions for paired-samples $t$ tests were evaluated by the project's statistician, who found them to be reasonably met for assessing pre-and postdietary intake, food security, and blood pressure. Participants experienced significant improvement in daily dietary fiber intake (mean, 14.0-17.1; $t=-4.110 ; P<.001)$ and a slight yet nonsignificant increase in daily fruit and vegetable intake (mean, 3.4-3.6 cups; $t=-1.597$; $P=.12$ ) compared with baseline. Mean food security did not change. Among participants who accessed food assistance at least 4 times and who had high blood pressure at enrollment $(\mathrm{n}=17)$, diastolic blood pressure significantly improved (mean, 90.9-83.9; $t=2.950 ; P=.009)$.

\section{DISCUSSION AND IMPLICATIONS FOR FUTURE PRACTICE}

This pilot program provided a framework for medically tailored food assistance that may be replicated by other clinics and food bank partners. Future interventions might incorporate formative evaluation measures to assess curriculum use, intervention food consumption, and barriers to ongoing participation. Preliminary outcomes indicate that the program may support meaningful eating behavior change and blood pressure management. Randomized control trials of food pharmacy interventions are needed to confirm these relationships.

\section{NOTES}

Approval for this research was granted by the University of Oklahoma Health Sciences Center Institutional Review Board. The curriculum and list of intervention foods are available upon request to the primary author. This project was funded by Blue Cross and Blue Shield of Oklahoma, the George Kaiser Family Foundation, and the Community Food Bank of Eastern Oklahoma. The authors thank Dr. Kara Beair-Butler for her early support of the University of Oklahoma Food Pharmacy.

\section{REFERENCES}

1. US Department of Agriculture, Economic Research Service. Definitions of food security. http://www.ers.usda.gov/ topics/food-nutrition-assistance/foodsecurity-in-the-us/definitions-of-foodsecurity.aspx. Accessed June 13, 2018.

2. American Diabetes Association. Improving care and promoting health in populations: Standards of Medical Care in Diabetes-2018. Diabetes Care. 2017;41 (suppl 1):S7-S12.

3. Seligman HK, Schillinger D. Hunger and socioeconomic disparities in chronic disease. N Engl J Med. 2010;363:6-9.

4. Barnidge E, Stenmark S, Seligman H. Clinic-to-community models to address food insecurity. JAMA Pediatr. 2017;171: 507-508.

5. Mello JA, Gans KM, Risica PM, Kirtania U, Strolla LO, Fournier L. How is food insecurity associated with dietary behaviors? An analysis with low income, ethnically diverse participants in a nutrition intervention study. J Am Diet Assoc. 2010;110:1906-1911.

6. Campbell AP. DASH eating plan: an eating pattern for diabetes management. Diabetes Spectr. 2017;30:76-81.

7. Block G, Gillespie C, Rosenbaum EH, Jenson C. A rapid food screener to assess fat and fruit and vegetable intake. $A m \mathrm{~J}$ Prev Med. 2000;18:284-288.

8. US Department of Agriculture, Economic Research Service. US Household Food Security Survey Module: Threestage design, with screeners. 2012. https://www.ers.usda.gov/media/8271/ hh2012.pdf. Accessed July 19, 2017.

9. Weinfield NS, Mills G, Borger C, et al. Hunger in America 2014. Rockville, MA: Westat and the Urban Institute; 2014. 